\title{
Convergence and Application of a Modified Iteratively Regularized Gauss-Newton Algorithm
}

\author{
Alexandra Smirnova* $^{*} \quad$ Rosemary A Renaut ${ }^{\dagger} \quad$ Taufiquar Khan ${ }^{\ddagger}$
}

February 13, 2007

\begin{abstract}
We establish theoretical convergence results for an Iteratively Regularized Gauss Newton (IRGN) algorithm with a specific Tikhonov regularization. This Tikhnov regularization, which uses a seminorm generated by a linear operator, is motivated by mapping of the minimization variables to physical space which exposes the different scales of the parameters and therefore also suggests appropriate weighting of the regularization terms with respect to the parameter spaces. The basic convergence result uses an a posteriori stopping rule and a modified source condition, without any restriction on the nonlinearity of the operator. We illustrate our theoretical results using simulations for a one dimensional version of the exponentially ill-posed optical tomography inverse problem for which the parameter space depends on diffusion coefficient $D$ and absorption coefficient $\mu$ which are on very different scales. We conclude that the new method contributes greater flexibility for implementations of IRGN solutions of ill-posed inverse problems in which differing scales in physical space hinder standard IRGN inversions.
\end{abstract}

Keywords: Gauss-Newton method, discrepancy principle, ill-posed problem, regularization, optical tomography.

AMS Subject Classification: 47A52, 65F22, 65J15, 65N21.

\section{Introduction}

The iteratively Gauss-Newton method (IRGN) for the solution of exponentially ill-posed inverse problems, which was introduced by Bakushinsky in 1993 [BA93], has been investigated and extended in a number of formulations, [BK01, BK04, BS05, BKA06, K00]. Here, our goal is to extend the IRGN algorithm to nonlinear problems in which physical considerations suggest replacement of the stabilizing term of the IRGN by a more general Tikhonov

\footnotetext{
*Georgia State University, Department of Mathematics and Statistics, Atlanta, GA 30303-3083. Tel: 404-651-0641, Fax: 404-651-2246.

${ }^{\dagger}$ Supported by NSF grants DMS 0513214 and DMS 0421846. Arizona State University, Department of Mathematics and Statistics, Tempe, AZ 85287-1804. Tel: 480-965-3785, Fax: 480-965-4160.

${ }^{\ddagger}$ Clemson University, Department of Mathematical Sciences, Clemson, SC 29634-0975. Tel: 864-656-3257, Fax: 864-656-5230
} 
penalty stabilizing term. The particular study was motivated by solution of the optical tomography problem in one dimension, previously investigated in [KS05, BKS05], for which the mapping of the coefficient space to physical space highlights the different scales of the diffusion and absorption coefficients which had prevented their simultaneous recovery, but for which the new formulation is successful.

\subsection{Formulation}

Assume that a nonlinear operator $F$ acts on a pair of Hilbert spaces $\left(\mathbf{H}, \mathbf{H}_{\mathbf{1}}\right)$, that is $F: \mathbf{H} \rightarrow \mathbf{H}_{\mathbf{1}}$, and that, $F$ is Fréchet differentiable in $\mathbf{H}$ without such structural assumptions as monotonicity, or invertibility of $F^{* *}(\cdot) F^{\prime}(\cdot)$, etc. Let the following conditions hold:

$$
\begin{gathered}
\left\|F^{\prime}\left(\mathbf{q}_{1}\right)\right\| \leq M_{1} \quad \text { for any } \quad \mathbf{q}_{1} \in \mathbf{H} \\
\left\|F^{\prime}\left(\mathbf{q}_{1}\right)-F^{\prime}\left(\mathbf{q}_{2}\right)\right\| \leq M_{2}\left\|\mathbf{q}_{1}-\mathbf{q}_{2}\right\| \quad \text { for any } \quad \mathbf{q}_{1}, \mathbf{q}_{2} \in \mathbf{H},
\end{gathered}
$$

and $\mathcal{F}\left(M_{1}, M_{2}\right)$ be the class of operators $F$ satisfying (1.1) and (1.2).

We consider the general problem of minimizing the functional

$$
J(\mathbf{q}):=\left\|F(\mathbf{q})-g_{\delta}\right\|_{\mathbf{H}_{1}}^{2}
$$

where $g_{\delta}$ approximates the exact data $g$ with the accuracy $\delta$, i.e.,

$$
\left\|g-g_{\delta}\right\| \leq \delta
$$

Of primary interest is the case in which there exists exact data for which functional (1.3) is identically zero, i.e., there is some element $\hat{\mathbf{q}} \in \mathbf{H}$ (maybe nonunique) such that

$$
\|F(\hat{\mathbf{q}})-g\|_{\mathbf{H}_{\mathbf{1}}}=\inf _{\mathbf{q} \in \mathbf{H}}\|F(\mathbf{q})-g\|_{\mathbf{H}_{\mathbf{1}}}=0 .
$$

Suppose $\left\{\tau^{(k)}\right\}$ is some sequence of regularization parameters satisfying the conditions

$$
\tau^{(k)} \geq \tau^{(k+1)}>0, \quad \sup _{k \in \mathcal{N} \cup 0} \frac{\tau^{(k)}}{\tau^{(k+1)}}=d<\infty, \quad \lim _{k \rightarrow \infty} \tau^{(k)}=0,
$$

$\mathbf{q}^{(k)}$ is the current point of the iterative process we are trying to construct and Tikhonov's functional is of the following form

$$
J_{\tau^{(k)}}(\mathbf{q})=\frac{1}{2}\left\|F(\mathbf{q})-g_{\delta}\right\|_{\mathbf{H}_{\mathbf{1}}}^{2}+\frac{\tau^{(k)}}{2}\|L(\mathbf{q}-\overline{\mathbf{q}})\|_{\mathbf{H}_{\mathbf{2}}}^{2} .
$$

Here $L$ is a linear operator from a Hilbert space $\mathbf{H}$ to a Hilbert space $\mathbf{H}_{2}, \overline{\mathbf{q}} \in \mathbf{H}$, which is chosen appropriate to the underlying physical values for $\mathbf{q}$ and $\overline{\mathbf{q}}$ is in general a vector of constraining values for $\mathbf{q}$. Replacing the discrepancy term (1.3) with its approximation

$$
J\left(\mathbf{q}^{(k)} ; \mathbf{q}\right)=\frac{1}{2}\left\|F\left(\mathbf{q}^{(k)}\right)-g_{\delta}+F^{\prime}\left(\mathbf{q}^{(k)}\right)\left(\mathbf{q}-\mathbf{q}^{(k)}\right)\right\|_{\mathbf{H}_{\mathbf{1}}}^{2}
$$


provides, instead of $J_{\tau^{(k)}}$, a strongly convex quadratic functional

$$
J_{\tau^{(k)}}\left(\mathbf{q}^{(k)} ; \mathbf{q}\right)=\frac{1}{2}\left\|F\left(\mathbf{q}^{(k)}\right)-g_{\delta}+F^{\prime}\left(\mathbf{q}^{(k)}\right)\left(\mathbf{q}-\mathbf{q}^{(k)}\right)\right\|_{\mathbf{H}_{1}}^{2}+\frac{\tau^{(k)}}{2}\|L(\mathbf{q}-\overline{\mathbf{q}})\|_{\mathbf{H}_{2}}^{2}, \quad \mathbf{q} \in \mathbf{H} .
$$

Its unique global minimum, denoted by $\tilde{\mathbf{q}}^{(\mathbf{k})}$, assuming necessary invertibility conditions is given explicitly by

$$
\begin{gathered}
\tilde{\mathbf{q}}^{(\mathbf{k})}=\mathbf{q}^{(k)}-\left[F^{\prime *}\left(\mathbf{q}^{(k)}\right) F^{\prime}\left(\mathbf{q}^{(k)}\right)+\tau^{(k)} L^{*} L\right]^{-1}\left\{F^{\prime *}\left(\mathbf{q}^{(k)}\right)\left(F\left(\mathbf{q}^{(k)}\right)-g_{\delta}\right)\right. \\
\left.+\tau^{(k)} L^{*} L\left(\mathbf{q}^{(k)}-\overline{\mathbf{q}}\right)\right\} .
\end{gathered}
$$

This usage of an arbitrary linear operator $L$ in the penalty term can be very beneficial for some inverse problems. As is standard in the optimization literature, [NW99], we generalize the algorithm one step further by use of a line search procedure, through the introduction of a variable step size $\alpha^{(k)}$ such that

$$
0<\alpha \leq \alpha^{(k)} \leq 1
$$

yielding our following modification of the IRGN algorithm:

$$
\begin{gathered}
\mathbf{q}^{(k+1)}=\mathbf{q}^{(k)}-\alpha^{(k)}\left[F^{\prime *}\left(\mathbf{q}^{(k)}\right) F^{\prime}\left(\mathbf{q}^{(k)}\right)+\tau^{(k)} L^{*} L\right]^{-1}\left\{F^{\prime *}\left(\mathbf{q}^{(k)}\right)\left(F\left(\mathbf{q}^{(k)}\right)-g_{\delta}\right)\right. \\
\left.+\tau^{(k)} L^{*} L\left(\mathbf{q}^{(k)}-\overline{\mathbf{q}}\right)\right\}
\end{gathered}
$$

Due to the inexact nature of $g_{\delta}$ the iterations are terminated early using an a posteriori stopping rule. Here we adopt the stopping rule presented in [BS05] and terminate the iterations at the first index $\mathcal{K}=\mathcal{K}(\delta)$, for which the residual $\left\|F\left(\mathbf{q}^{(k)}\right)-g_{\delta}\right\|$ is less than or equal to $\sqrt{\rho \delta}, \rho>1$ :

$$
\left\|F\left(\mathbf{q}^{(\mathcal{K})}\right)-g_{\delta}\right\|^{2} \leq \rho \delta<\left\|F\left(\mathbf{q}^{(k)}\right)-g_{\delta}\right\|^{2}, \quad 0 \leq k \leq \mathcal{K}, \quad \rho>1 .
$$

Regarding $\tilde{\mathbf{q}}^{(\mathbf{k})}$ in (1.10) as the next element of the iterative process and taking $L=I$ yields the original stabilizing IRGN method, see [BA93, BK04]. The convergence analysis of IRGN in [BA93] was done under the source type condition:

$$
\hat{\mathbf{q}}-\overline{\mathbf{q}} \in \varphi\left(F^{\prime *}(\hat{\mathbf{q}}) F^{\prime}(\hat{\mathbf{q}})\right) S, \quad S:=\{\mathbf{v} \in \mathbf{H},\|\mathbf{v}\| \leq \text { const }\} .
$$

In [BA97] and [BK01] for $\varphi(t)=t^{p}, p \geq \frac{1}{2}$, it is shown that

$$
\left\|\mathbf{q}^{(\mathcal{K})}-\hat{\mathbf{q}}\right\|=0\left(\delta^{\frac{2 p}{2 p+1}}\right),
$$

if $\mathcal{K}=\mathcal{K}(\delta)$ is chosen by the a priori stopping rule:

$$
\delta \sim\left(\tau^{(\mathcal{K})}\right)^{p+\frac{1}{2}}
$$

In [BNS97], on the other hand, the following rather restictive conditions were imposed on $F^{\prime}$

$$
F^{\prime}\left(\mathbf{q}_{1}\right)=R\left(\mathbf{q}_{1}, \mathbf{q}_{2}\right) F^{\prime}\left(\mathbf{q}_{2}\right)+Q\left(\mathbf{q}_{1}, \mathbf{q}_{2}\right)
$$




$$
\begin{gathered}
\left\|I-R\left(\mathbf{q}_{1}, \mathbf{q}_{2}\right)\right\| \leq C_{R} \\
\left\|Q\left(\mathbf{q}_{1}, \mathbf{q}_{2}\right)\right\| \leq C_{Q}\left\|F^{\prime}(\hat{\mathbf{q}})\left(\mathbf{q}_{1}-\mathbf{q}_{2}\right)\right\|, \quad \mathbf{q}_{1}, \mathbf{q}_{2} \in B_{\sigma}(\hat{\mathbf{q}})
\end{gathered}
$$

where $\sigma, C_{R}, C_{Q}$ are assumed to be sufficiently small. Then the following convergence rates were obtained for $\varphi(t)=t^{p}, 0 \leq p \leq \frac{1}{2}$

$$
\left\|\mathbf{q}^{(\mathcal{K})}-\hat{\mathbf{q}}\right\|:= \begin{cases}\mathrm{o}\left(\delta^{\frac{2 p}{2 p+1}}\right), & \text { if } 0 \leq p<\frac{1}{2}, \\ 0(\sqrt{\delta}), & p=\frac{1}{2},\end{cases}
$$

provided that $\mathcal{K}$ is chosen by the more generous a posteriori stopping rule

$$
\left\|F\left(\mathbf{q}^{(\mathcal{K})}\right)-g_{\delta}\right\| \leq \rho \delta<\left\|F\left(\mathbf{q}^{(k)}\right)-g_{\delta}\right\|, \quad 0 \leq k \leq \mathcal{K}, \quad \rho>1
$$

Under the same stringent conditions on $F^{\prime}$, but with the following source function

$$
\varphi(t)= \begin{cases}\left(-\ln \frac{t}{e t_{0}}\right)^{-p} & \text { if } 0<t \leq t_{0} \\ 0 & \text { if } t=0\end{cases}
$$

in (1.12), where $t_{0} \geq\left\|F^{\prime}(\cdot)\right\|^{2}, p>0$, and $e$ is Euler's constant, logarithmic convergence rates were derived under assumption (1.15) in [H97].

An attractive feature of our convergence theorem for algorithm (1.10)-(1.11) is that it does not require nonlinearity condition (1.15), which is not satisfied for many practically important inverse problems, including the Diffusion Optical Tomography (DOT) inverse problem [A99], discussed in Section 3. In particular we impose the source condition used in [KW93]:

$$
L^{*} L(\hat{\mathbf{q}}-\overline{\mathbf{q}}) \in F^{*}(\hat{\mathbf{q}}) S, \quad S:=\{\mathbf{v} \in \mathbf{H},\|\mathbf{v}\| \leq \varepsilon\}
$$

which is equivalent to (1.12) for $p=\frac{1}{2}$ with $\varphi(t)=t^{p}$ when $L=I$. Additionally, the use of an arbitrary linear operator $L$ in place of the identity operator in the IRGN method makes it possible for the regularization to be imposed in an alternative space. This proves to be extremely beneficial for the DOT inverse problem in which regularization is applied directly in physical space rather than in the B-spline coefficient space, (see Section 4), and for which the mapping exposes the impact of the different sensitivities with respect to each parameter.

The remainder of the paper is as follows. In Section 2 we present and prove our main theorem for the convergence of (1.10). In Section 3 we briefly present the numerical experiments designed to evaluate the theory of Section 2, greater detail already having been provided in other references, [KS05, BKS05]. Numerical results are presented in Section 4 and final conclusions given in Section 5.

\section{Convergence Theorem}

We now formulate our basic convergence result for iteratively regularized algorithm (1.10) combined with stopping rule (1.11). 


\section{Theorem I}

Assume that

1. $F$ is in the class $\mathcal{F}\left(M_{1}, M_{2}\right)$; conditions (1.4) and (1.5) hold.

2. The regularization sequence $\left\{\tau^{(k)}\right\}$ and the step size sequence $\left\{\alpha^{(k)}\right\}$ are chosen according to (1.6) and (1.9) respectively.

3. The source condition (1.19) is satisfied.

4. The linear operator $L^{*} L$ is surjective and there is a constant $m>0$ such that

$$
\left(L^{*} L \mathbf{h}, \mathbf{h}\right) \geq m\|\mathbf{h}\|^{2} .
$$

5. Constants defining $F$ and the iteration are constrained by

$$
\begin{gathered}
\frac{M_{2} \varepsilon}{m}+\frac{d-1}{d \alpha}+\sqrt{\frac{\varepsilon}{m}\left\{\frac{M_{2}}{2}+\frac{M_{1}^{2}}{(\sqrt{\rho}-1)^{2}}\right\}} \leq 1, \\
\frac{\left\|\mathbf{q}^{(0)}-\hat{\mathbf{q}}\right\|}{\sqrt{\tau^{(0)}}} \leq \frac{\varepsilon}{\sqrt{m}\left\{1-\frac{M_{2} \varepsilon}{m}-\frac{d-1}{d \alpha}\right\}}:=l .
\end{gathered}
$$

Then

1. For iterations (1.10)

$$
\frac{\left\|\mathbf{q}^{(k)}-\hat{\mathbf{q}}\right\|}{\sqrt{\tau^{(k)}}} \leq l, \quad k=0,1, \ldots, \mathcal{K}(\delta),
$$

where $\mathcal{K}=\mathcal{K}(\delta)$ is calculated by a posteriori rule (1.11).

2. The sequence $\{\mathcal{K}(\delta)\}$ is admissible, i.e.

$$
\lim _{\delta \rightarrow 0}\left\|\mathbf{q}^{(\mathcal{K}(\delta))}-\mathbf{z}\right\|=0
$$

$\mathbf{z}$ is $\operatorname{arginf}_{\mathbf{q} \in \mathbf{H}}\|F(\mathbf{q})-g\|_{\mathbf{H}_{\mathbf{1}}}$.

Proof of Theorem I Since $L^{*} L$ is surjective and

$$
\left(\left[F^{\prime *}(\mathbf{q}) F^{\prime}(\mathbf{q})+\tau L^{*} L\right] \mathbf{h}, \mathbf{h}\right) \geq \tau m\|\mathbf{h}\|^{2}
$$

for arbitrary $\tau>0, \mathbf{q}, \mathbf{h} \in \mathbf{H}$, the operator $\left[F^{\prime *}(\mathbf{q}) F^{\prime}(\mathbf{q})+\tau L^{*} L\right]^{-1}$ exists,

$$
\left\|\left[F^{* *}(\mathbf{q}) F^{\prime}(\mathbf{q})+\tau L^{*} L\right]^{-1}\right\| \leq \frac{1}{\tau m}
$$

and iterations (1.10) are well-defined.

Suppose for any $j, 0 \leq j \leq k<\mathcal{K}(\delta)$, the induction assumption is fulfilled:

$$
\sigma_{j}:=\frac{\left\|\mathbf{q}^{(j)}-\hat{\mathbf{q}}\right\|}{\sqrt{\tau^{(j)}}} \leq l .
$$


Then by (1.5)

$$
\begin{gathered}
F\left(\mathbf{q}^{(k)}\right)-g_{\delta}=F^{\prime}\left(\mathbf{q}^{(k)}\right)\left(\mathbf{q}^{(k)}-\hat{\mathbf{q}}\right)-\mathcal{B}\left(\mathbf{q}^{(k)}, \hat{\mathbf{q}}\right)\left(\mathbf{q}^{(k)}-\hat{\mathbf{q}}\right)\left(\mathbf{q}^{(k)}-\hat{\mathbf{q}}\right)+g-g_{\delta}, \\
\left\|\mathcal{B}\left(\mathbf{q}^{(k)}, \hat{\mathbf{q}}\right)\right\| \leq \frac{M_{2}}{2}
\end{gathered}
$$

Inequality (2.7) and condition (1.19) imply

$$
\begin{gathered}
\mathbf{q}^{(k+1)}-\hat{\mathbf{q}}=\mathbf{q}^{(k)}-\hat{\mathbf{q}}-\alpha^{(k)}\left[F^{\prime *}\left(\mathbf{q}^{(k)}\right) F^{\prime}\left(\mathbf{q}^{(k)}\right)+\tau^{(k)} L^{*} L\right]^{-1}\left[F^{\prime *}\left(\mathbf{q}^{(k)}\right) F^{\prime}\left(\mathbf{q}^{(k)}\right)+\tau^{(k)} L^{*} L\right]\left(\mathbf{q}^{(k)}-\hat{\mathbf{q}}\right) \\
-\alpha^{(k)}\left[F^{\prime *}\left(\mathbf{q}^{(k)}\right) F^{\prime}\left(\mathbf{q}^{(k)}\right)+\tau^{(k)} L^{*} L\right]^{-1} F^{\prime *}\left(\mathbf{q}^{(k)}\right)\left\{-\mathcal{B}\left(\mathbf{q}^{(k)}, \hat{\mathbf{q}}\right)\left(\mathbf{q}^{(k)}-\hat{\mathbf{q}}\right)\left(\mathbf{q}^{(k)}-\hat{\mathbf{q}}\right)+g-g_{\delta}+\tau^{(k)} \mathbf{v}\right\} \\
-\alpha^{(k)} \tau^{(k)}\left[F^{\prime *}\left(\mathbf{q}^{(k)}\right) F^{\prime}\left(\mathbf{q}^{(k)}\right)+\tau^{(k)} L^{*} L\right]^{-1}\left\{F^{\prime}\left(\mathbf{q}^{(k)}\right)-F^{\prime}(\hat{\mathbf{q}})\right\}^{*} \mathbf{v}
\end{gathered}
$$

To estimate $\left\|\left[F^{\prime *}\left(\mathbf{q}^{(k)}\right) F^{\prime}\left(\mathbf{q}^{(k)}\right)+\tau^{(k)} L^{*} L\right]^{-1} F^{\prime *}\left(\mathbf{q}^{(k)}\right)\right\|$, we note that for any bounded linear operator in a Hilbert space a polar decomposition holds. Hence

$$
F^{\prime}\left(\mathbf{q}^{(k)}\right)=U\left|F^{\prime}\left(\mathbf{q}^{(k)}\right)\right|
$$

where $\left|F^{\prime}\left(\mathbf{q}^{(k)}\right)\right|:=\left(F^{\prime *}\left(\mathbf{q}^{(k)}\right) F^{\prime}\left(\mathbf{q}^{(k)}\right)\right)^{1 / 2}$ and $U$ is a partial isometry:

$$
\left\|U \mathbf{q}_{1}\right\|=\left\|\mathbf{q}_{1}\right\| \quad \forall \mathbf{q}_{1} \in \mathcal{N}(U)^{\perp}, \quad \mathcal{N}(U):=\left\{\mathbf{q}_{1}: U \mathbf{q}_{1}=0\right\} .
$$

Now, denoting

$$
A^{(k)}:=F^{* *}\left(\mathbf{q}^{(k)}\right) F^{\prime}\left(\mathbf{q}^{(k)}\right), \quad B=L^{*} L . \quad \text { and } \quad C^{(k)}:=\left(A^{(k)}\right)^{1 / 2} B^{-1 / 2},
$$

condition (2.1) yields

$$
\begin{gathered}
\left\|\left[F^{\prime *}\left(\mathbf{q}^{(k)}\right) F^{\prime}\left(\mathbf{q}^{(k)}\right)+\tau^{(k)} L^{*} L\right]^{-1} F^{\prime *}\left(\mathbf{q}^{(k)}\right)\right\|=\left\|\left[A^{(k)}+\tau^{(k)} B\right]^{-1}\left(A^{(k)}\right)^{1 / 2} U^{*}\right\| \leq\left\|\left[A^{(k)}+\tau^{(k)} B\right]^{-1}\left(A^{(k)}\right)^{1 / 2}\right\| \\
=\left\|\left[B^{1 / 2}\left(B^{-1 / 2} A^{(k)} B^{-1 / 2}+\tau^{(k)} I\right) B^{1 / 2}\right]^{-1}\left(A^{(k)}\right)^{1 / 2}\right\|=\left\|B^{-1 / 2}\left(\left(C^{(k)}\right)^{*} C^{(k)}+\tau^{(k)} I\right)^{-1}\left(C^{(k)}\right)^{*}\right\| \\
\quad \leq\left\|B^{-1 / 2}\right\|\left\|\left(\left(C^{(k)}\right)^{*} C^{(k)}+\tau^{(k)} I\right)^{-1}\left(\left(C^{(k)}\right)^{*} C^{(k)}\right)^{1 / 2}\right\| \leq \frac{1}{\sqrt{m}} \max _{t \geq 0} \frac{\sqrt{t}}{t+\tau^{(k)}} \leq \frac{1}{2 \sqrt{\tau^{(k)} m}}
\end{gathered}
$$

Combining (2.8) and (2.9) one derives

$$
\begin{gathered}
\left\|\mathbf{q}^{(k+1)}-\hat{\mathbf{q}}\right\| \leq\left(1-\alpha^{(k)}\right)\left\|\mathbf{q}^{(k)}-\hat{\mathbf{q}}\right\|+\frac{\alpha^{(k)}}{2 \sqrt{\tau^{(k)} m}}\left\{\left.\frac{M_{2}}{2}\left\|\mathbf{q}^{(k)}-\hat{\mathbf{q}}\right\|\right|^{2}+\delta+\tau^{(k)}\|\mathbf{v}\|\right\} \\
+\frac{\alpha^{(k)} M_{2}}{m}\left\|\mathbf{q}^{(k)}-\hat{\mathbf{q}}\right\|\|\mathbf{v}\| .
\end{gathered}
$$

Also, for $k<\mathcal{K}(\delta)$, according to (1.11)

$$
\rho \delta \leq\left\|F\left(\mathbf{q}^{(k)}\right)-g_{\delta}\right\|^{2} .
$$

Thus, by (1.1)

$$
\sqrt{\rho \delta} \leq\left\|F\left(\mathbf{q}^{(k)}\right)-g\right\|+\left\|g-g_{\delta}\right\| \leq M_{1}\left\|\mathbf{q}^{(k)}-\hat{\mathbf{q}}\right\|+\delta,
$$


and

$$
\sqrt{\rho \delta}-\delta \leq M_{1}\left\|\mathbf{q}^{(k)}-\hat{\mathbf{q}}\right\|
$$

Without loss of generality one can assume $\delta<1$, yielding

$$
\delta \leq \frac{M_{1}^{2}\left\|\mathbf{q}^{(k)}-\hat{\mathbf{q}}\right\|^{2}}{(\sqrt{\rho}-1)^{2}} .
$$

Hence

$$
\begin{gathered}
\left\|\mathbf{q}^{(k+1)}-\hat{\mathbf{q}}\right\| \leq\left(1-\alpha^{(k)}+\frac{\alpha^{(k)} M_{2} \varepsilon}{m}\right)\left\|\mathbf{q}^{(k)}-\hat{\mathbf{q}}\right\| \\
+\frac{\alpha^{(k)}}{2 \sqrt{\tau^{(k)} m}}\left\{\frac{M_{2}}{2}+\frac{M_{1}^{2}}{(\sqrt{\rho}-1)^{2}}\right\}\left\|\mathbf{q}^{(k)}-\hat{\mathbf{q}}\right\|^{2}+\frac{\alpha^{(k)} \sqrt{\tau^{(k)}} \varepsilon}{2 \sqrt{m}} .
\end{gathered}
$$

Conditions (1.6) and (1.19) together with induction assumption (2.6) yield

$$
\sigma_{k+1} \leq\left(1-\alpha^{(k)}+\frac{\alpha^{(k)} M_{2} \varepsilon}{m}\right) d l+\frac{\alpha^{(k)} l^{2} d}{2 \sqrt{m}}\left\{\frac{M_{2}}{2}+\frac{M_{1}^{2}}{(\sqrt{\rho}-1)^{2}}\right\}+\frac{\alpha^{(k)} d \varepsilon}{2 \sqrt{m}} .
$$

From (1.9), (2.15), (2.2) and (2.3) it follows that $\sigma_{k+1} \leq l$.

The sequence $\mathcal{K}=\mathcal{K}(\delta)$ is nondecreasing as $\delta \rightarrow 0$. Two cases are possible:

1. $\mathcal{K}(\delta)=\mathcal{K}_{0}$ for any $\delta \leq \delta_{0}$. Then by (1.4) and (1.11) $\lim _{\delta \rightarrow 0} \mathbf{q}^{\left(\mathcal{K}_{0}\right)}\left(g_{\delta}\right)$ is $\operatorname{arginf}_{\mathbf{q} \in \mathbf{H}}\|F(\mathbf{q})-g\|_{\mathbf{H}_{1}}^{2}$.

2. $\mathcal{K}(\delta) \rightarrow \infty$ as $\delta \rightarrow 0$. Then

$$
\left\|\mathbf{q}^{(\mathcal{K}(\delta))}-\hat{\mathbf{q}}\right\| \leq l \sqrt{\tau^{(\mathcal{K}(\delta))}} \rightarrow 0 \quad \text { as } \quad \delta \rightarrow 0
$$

In either case $\mathbf{q}^{(\mathcal{K}(\delta))}$ converges in the norm of $\mathbf{H}$ to $\operatorname{arginf}_{\mathbf{q} \in \mathbf{H}}\|F(\mathbf{q})-g\|_{\mathbf{H}_{1}}$ as $\delta \rightarrow 0$, hence completing the proof.

\section{Numerical Application}

Optical tomography is a way to probe highly scattering media using low-energy visible or near infra-red light so as to reconstruct images of these media. Light in the near-infrared range (wavelength from 700 to $1200 \mathrm{~nm}$ ) penetrates and interacts with tissue, with predominant effects being absorption and scattering [DC97, HAD97, CA93]. The widely accepted photon transport model is the radiative transfer equation (RTE), which is an integro-differential equation for the radiance and has spatially dependent diffusion and absorption parameters as coefficients which are a priori unknown. Hence the inverse problem is to infer from the measurements of the photon density on the boundary, the coefficients of absorption and diffusion within the tissue. A low order Diffusion Approximation (DA) to the RTE, which yields a parabolic differential equation in the time dependent case and an elliptic equation for steady state, has been derived and studied in the last several years, [A99]. This DA has been widely used to calculate photon migration in biological tissues [HAB98], and the existing computational methods for the inverse problem of photon migration in biological tissues are almost exclusively based on the DA [CA95]. 
It is well known that the DOT inverse problem is exponentially ill-posed or unstable [NW01, A99]. In fact, the one dimensional version of the DOT ill-posed inverse problem was studied recently using the IRGN method [KS05, BKS05], and it was limitations in these studies which motivated our presented modification of the IRGN (1.10). We therefore illustrate the performance of (1.10) for yielding improved solutions of the DOT inverse problem.

\subsection{Problem Formulation}

The inverse problem of optical tomography, using the diffusion approximation to the radiative transfer model, requires the estimation of the underlying model parameters $\mathbf{q}$, the coefficient of diffusion $D$ and the coefficient of absorption $\mu$ i.e. $\mathbf{q}=(D, \mu)^{T}$, given measured values of the density $u$ on the boundary.

The photon density $u$ solves the steady state parameter dependent equation

$$
-\nabla \cdot D(\mathbf{x}) \nabla u(\mathbf{x})+\mu(\mathbf{x}) u(\mathbf{x})=s(\mathbf{x}),
$$

with boundary conditions

$$
u(\mathbf{x} ; \mathbf{q})+2 D(\mathbf{x}) \frac{\partial u(\mathbf{x})}{\partial \nu}=0, \quad \mathbf{x} \in \partial \Omega .
$$

Here $\mathrm{x}$ is the spatial variable on the domain $\Omega, \nu$ is the normal direction from the boundary and $s$ represents the forcing function, or source. A finite element Galerkin method using B-spline expansions for each of $u(\mathbf{x}), D(\mathbf{x})$ and $\mu(\mathbf{x})$ for the solution of the forward problem is described in detail in [BKS05, KS05]. Given the B-spline expansions for $D$ and $\mu$, with coefficients $\left(q_{D}\right)_{l}, l=1 \ldots N_{D}$ and $\left(q_{\mu}\right)_{l}, l=1 \ldots N_{\mu}$, the expansion coefficients $\left(q_{u}\right)_{l}, l=1 \ldots N_{u}$ for $u$ are obtained as the solution of the linear system of equations which is obtained through the variational formulation applied to $(3.1,3.2)$. Solving subject to multiple sources $s_{j}, j=1 \ldots n_{s}$, yields a set of observables for $u$ on the boundary, denoted by values $g_{i j}, i=1 \ldots n_{m}$, where $n_{m}$ is the number of measurements. The coefficients of $u$, and hence the boundary values $g_{i j}$, are explicitly dependent on the parameter vector of B-spline coefficients for $D$ and $\mu, \mathbf{q}^{T}=\left(\mathbf{q}_{D}{ }^{T}, \mathbf{q}_{\mu}{ }^{T}\right)$.

In simulating the solution of the inverse problem for optical tomography, with unknown $D$ and $\mu$, we solve the forward problem $(3.1,3.2)$ to high accuracy to yield measurement values $g_{\delta}$. Then the same forward problem is solved using an alternative discretization yielding observables $C_{i j}$ which approximate $g_{\delta_{i j}}$. The $C_{i j}$ depend (nonlinearly) on the unknown model parameters $\mathbf{q} \in \mathcal{R}^{N}$, where $N=N_{\mu}+N_{D}$. This inverse problem can be cast as a finite dimensional minimization problem

$$
\begin{aligned}
\frac{1}{2} \arg \min _{\mathbf{q} \in \mathcal{R}^{N}} J(\mathbf{q}) & =\frac{1}{2} \arg \min _{\mathbf{q}} \sum_{j=1}^{n_{m}} \sum_{i=1}^{n_{s}}\left(C_{i j}(u(\mathbf{q}))-g_{i j}\right)^{2} . \\
& =\frac{1}{2} \arg \min _{\mathbf{q}}\|R(\mathbf{q})\|^{2} .
\end{aligned}
$$

Here the residual vector $R \in \mathcal{R}^{n}, n=n_{s} \times n_{m}$, comprises all terms $C_{i j}-g_{\delta_{i j}}$. 
The discrete sensititivity relations presented in [BKS05, KS05] lead to an explicit expression for $K(\mathbf{q})$ which is the Jacobian matrix for $R$, yielding $\nabla J=K^{*}(\mathbf{q}) R(\mathbf{q})$, and Hessian which is approximated by the first order term only, $\nabla^{2} J(\mathbf{q}) \approx K^{*}(\mathbf{q}) K(\mathbf{q})$.

\subsection{Algorithms}

Given the Jacobian $K$, the standard iteratively regularized Gauss Newton method (IRGN) in the presence of noise in the measured values, [BA93, BKS05, KS05], is given by

$$
\mathbf{q}^{(k+1)}=\mathbf{q}^{(k)}+\alpha^{(k)} \mathbf{p}^{(k)} .
$$

where search direction $\mathbf{p}^{(k)}$ solves

$$
\left(K^{*}\left(\mathbf{q}^{(k)}\right) K\left(\mathbf{q}^{(k)}\right)+\tau^{(k)} I\right) \mathbf{p}^{(k)}=-\left(K^{*}\left(\mathbf{q}^{(k)}\right) R\left(\mathbf{q}^{(k)}\right)+\tau^{(k)}\left(\mathbf{q}^{(k)}-\overline{\mathbf{q}}\right)\right),
$$

Here $\tau^{(k)}$ is a regularizing sequence satisfying (1.6), and $\overline{\mathbf{q}}$ is a given reference value for $\mathbf{q}$.

In [BKS05] and [KS05] this IRGN was used to obtain estimates for $D(x)$ under the assumption that $\mu(x)$ was given. In our current experiments, both $\mu$ and $D$ are unknown and are to be reconstructed in the process of solving the inverse problem. To that end, in the following we illustrate two modifications of the IRGN in the context of determining the absorption and diffusion distributions in (3.1), both of which are based on physical considerations, and which are subject to the convergence theory presented in Theorem I.

On the one hand, the regularizing term $\|\mathbf{q}-\overline{\mathbf{q}}\|_{2}^{2}$ regularizes the solution in the B-spline coefficient space, whereas information on appropriate values for the absorption and diffusion are known directly in physical space. This suggests that the regularization be imposed directly in physical space and that the regularizing term in (3.5) be replaced by the more general Tikhonov regularizing term $\|L(\mathbf{q}-\overline{\mathbf{q}})\|_{2}^{2}$, where $L$ maps the coefficients to physical space. On the other hand, typical values of $D$ and $\mu$ for non-cancerous tissue are $D=.55$ and $\mu=.006$, with $D=.275$ and $\mu=.012$ for tumor; $D$ is between one and two orders of magnitude larger than $\mu$. Moreover, the analytic solution of the forward problem (3.1) with constant values of $D$ and $\mu$ depends explicitly on the ratio $w=D / \mu$. Specificially, the variations of $u$ with respect to $D$ and $\mu$, respectively, are proportional with weight $w$, and consequently the sensitivities of $J$ with respect to each variable are also on different scales. Therefore, the penalty term on $\mu$ should be appropriately weighted by $w$ in order to have an impact on regularizing the iterations.

In order to clarify these modifications, we rewrite (3.5) so as to emphasize the dependence on $\mathbf{q}_{D}$ and $\mathbf{q}_{\mu}$ individually, and to put the IRGN into the format appropriate for the Tikhonov functional given in (1.10). Specifically, decomposing $K$ as

$$
K=\nabla R=\left[\nabla_{\mathbf{q}_{D}} R, \nabla_{\mathbf{q}_{\mu}} R\right]=\left[K_{D}, K_{\mu}\right],
$$

with

$$
\nabla^{2} J(\mathbf{q}) \approx\left(\begin{array}{cc}
K_{D}^{*} K_{D} & K_{D}^{*} K_{\mu} \\
K_{\mu}^{*} K_{D} & K_{\mu}^{*} K_{\mu}
\end{array}\right)
$$


yields the block system for $\mathbf{p}^{(k)}$,

$$
\left[\left(\begin{array}{cc}
K_{D}^{*} K_{D} & K_{D}^{*} K_{\mu} \\
K_{\mu}^{*} K_{D} & K_{\mu}^{*} K_{\mu}
\end{array}\right)+\tau^{(k)} W\right]\left(\begin{array}{c}
\mathbf{p}_{D} \\
\mathbf{p}_{\mu}
\end{array}\right)=-\left(\begin{array}{c}
K_{D}^{*}\left(\mathbf{q}^{(k)}\right) R\left(\mathbf{q}^{(k)}\right) \\
K_{\mu}^{*}\left(\mathbf{q}^{(k)}\right) R\left(\mathbf{q}^{(k)}\right)
\end{array}\right)-\tau^{(k)} W\left(\begin{array}{c}
\left(\mathbf{q}_{D}-\overline{\mathbf{q}}_{D}\right) \\
\left(\mathbf{q}_{\mu}-\overline{\mathbf{q}}_{\mu}\right)
\end{array}\right)
$$

where

$$
W=\left[\begin{array}{cc}
L_{D}^{T} L_{D} & 0 \\
0 & w^{2} L_{\mu}^{T} L_{\mu}
\end{array}\right] .
$$

We refer to the case in which $L_{D}=I, L_{\mu}=I$, regularizing in the B-spline space, as the weighted IRGN, (WIRGN), and in which $L_{D}=B_{D}, L_{\mu}=B_{\mu}$, where in each case $B$ is the relevant mapping matrix to physical space from spline coefficient space, as Tikhonov weighted IRGN (TWIRGN), both of which are of the form suggested by (1.10), and assume that $L$ is block separable.

\subsection{Numerical Experiments}

A representative selection of experiments, with set up similar to those presented in [BKS05, KS05], which validate the performance of (1.10) for the optical tomography inverse problem in one dimension are presented. In each case the forward problem was solved using 153 linear basis functions for $u, D$ and $\mu$ on the domain $[0,43]$. There are a total of two detectors, $n_{m}=2$, at the boundaries and 10 sources, $n_{s}=10,5$ of which are equally spaced between .5 and 5.0 and the other 5 symmetrically placed at the other boundary.

In solving for the search direction in (3.5) at each step we use the equivalent least squares formulation, [NW99]. The line search parameter $\alpha^{(k)}$ is chosen through a backtracking strategy, $1,1 / 2, \ldots$, until the strong Wolfe conditions are satisfied, [NW99], or a maximum number of backtracking steps have been taken. Specifically, for cost functional $J, \alpha^{(k)}$ minimizes the scalar objective function

$$
\phi(\alpha)=J\left(\mathbf{q}^{(k)}+\alpha \mathbf{p}^{(k)}\right)
$$

under the constraints

$$
\begin{aligned}
J\left(\mathbf{q}^{(k)}+\alpha \mathbf{p}^{(k)}\right) & \leq J\left(\mathbf{q}^{(k)}\right)+\sigma_{1} \alpha \nabla J\left(\mathbf{q}^{(k)}\right)^{T} \mathbf{p}^{(k)} \\
\left|\nabla J\left(\mathbf{q}^{(k)}+\alpha \mathbf{p}^{(k)}\right)^{T} \mathbf{p}^{(k)}\right| & \leq-\sigma_{2} \nabla J\left(\mathbf{q}^{(k)}\right)^{T} \mathbf{p}^{(k)}
\end{aligned}
$$

Consistent with the experiments presented in [BKS05, KS05] using the IRGN to find solutions for (3.3), we use typical values $\sigma_{1}=.0001$ and $\sigma_{2}=.9$, [K99, BKS05]. Condition (2.2) in Theorem I imposes a necessary condition, $\alpha>(d-1) / d$, on the backtracking strategy relative to the chosen regularizing sequence for $\tau$. If the regularizing sequence decreases too quickly, no backtracking is possible. In the experiments with the IRGN we therefore compromise so as to permit at least 2 backtracking steps, $\alpha^{(k)}=1,0.5,0.25$ with $\tau^{(k)}$ chosen appropriately, $\tau^{(k)}=\tau^{(1)} c /(c+k-1), \tau^{(1)}=.025, c=4, d=1.25$. The initial choice $\tau^{(1)}$ is also effective in some cases for a Levenberg-Marquardt type implementation in which $L$ is chosen as for the IRGN methods, but the regularizing sequence on $\tau$ is held constant, $\tau^{(k)}=\tau^{(1)}$. Because condition (2.2) does not apply in this case, 
the number of backtracking steps is not limited and we therefore limit the total number to 6 . A further discussion of the choice of regularizing sequence for IRGN applied to this problem, in the case of known $\mu$ was provided in [BKS05, KS05].

Simulation with known parameters for $D$ and $\mu$ leads to a calculable residual of $\delta \approx 10^{-3}$ in (1.4), and therefore we use a stopping rule (1.11) with $\rho=5$. Although the number of iterations to reduce the residual further is recorded, the total number of search direction steps is restricted to 9 .

Inverse results using 101 basis functions for $u$, and 30 for each of $D$ and $\mu$ are given, although the algorithm also succeeds using $N_{D} \neq N_{\mu}$. Condition (2.1) imposes a bound on the finite dimensional operator $L$ in terms of calculable parameter $m=\min \sigma_{i}^{2}$, which in turn enters in (2.2) through its inverse, where $\sigma_{i}$ are the singular values of $L$ ordered from largest to smallest. For the B-spline coefficient matrices $1 / m$ decreases with the number of basis functions for $D$ and $\mu$, from roughly 91 for 10 basis functions to near 9 for 30 basis functions, and is larger when using the cubic bases as compared to linear, roughly 3343 and 319, respectively. It is also constant for weight parameter $w \geq 1$. Therefore the increase of $1 / \mathrm{m}$ from linear to cubic bases imposes far more severe restrictions on the other parameters within $(2.2,2.3)$ which depend not on the algorithm but on the underlying function $F$. For each inverse problem we thus solve using both linear and cubic spline bases for $D$ and $\mu$, and with regularization applied both in physical and coefficient space. Each formulation is also solved by the LM type method in order to show the effect of the regularizing sequence on the solution.

We present results for two different physical conditions for a tumor located between 1 and 6 on the domain $x \in[0,43]$, with tumor values $D=.275, \mu=.018$ and $D=.275, \mu=.012$, and background values $D=.55$, $\mu=.006$. Weight $w=D / \mu$ for cancer values was chosen, but an arbitrary choice, say, $w=25$ yields comparable results, while $w=1$ yields no converged results in any case tested.

\section{Experimental Results}

The results are detailed in Table 1 and Figures 1-2.

It is immediate from the results presented in Table 1 that the regularizing matrix used in the physical domain immediately impacts the convergence rate, and in particular that iteration using regularization in coefficient space for cubic splines is much less likely to converge, because of the lack of direct scaling relationship between $B$ and $I$ in this case. In any case where the Wolfe conditions were not satisfied within the limit of the backtracking steps, a steepest descent was taken and the line search initiated again. This accounts for the reported cases with more than a total of the maximum function evaluations expected for the given number of backtracking steps allowed, 27 and 63 for IRGN and LM, methods resp. It also accounts for case B with IRGN using $L=I$ in which quick convergence of the residual to below .005 occurs in two steps, but attempting to reduce the residual below .0001 is unsuccesful with just 2 backtracking steps and the steepest descent actually increases the residual. On the other hand, in this case if one permits 5 backtracking steps, violating condition (2.2) the residual is reduced to .00093 in 9 steps with 


\begin{tabular}{|c|c|c|c|c|c|c|c|c|c|}
\hline Problem & Method & $\begin{array}{c}\text { Regularizing } \\
\text { Matrix }\end{array}$ & Spline & $\begin{array}{c}\text { Steps } \\
5 \times 10^{-3}\end{array}$ & $\mathrm{FE}$ & $\begin{array}{c}\text { Residual } \\
\times 10^{-5}\end{array}$ & $\begin{array}{l}\text { Steps } \\
10^{-4}\end{array}$ & $\mathrm{FE}$ & $\begin{array}{c}\text { Residual } \\
\times 10^{-5}\end{array}$ \\
\hline $\mathrm{A}$ & IR & B & Linear & 3 & 6 & 20 & 9 & 50 & 16 \\
\hline $\bar{A}$ & IR & B & Cubic & 3 & 6 & 24 & 9 & 54 & 12 \\
\hline $\bar{A}$ & IR & $\mathrm{I}$ & Linear & 5 & 26 & 490 & 9 & 54 & 352 \\
\hline $\mathrm{A}$ & IR & I & Cubic & $*$ & $*$ & $*$ & 9 & 54 & 2964 \\
\hline $\mathrm{A}$ & LM & $\bar{B}$ & Linear & 3 & 6 & 41 & 9 & 48 & 37 \\
\hline $\mathrm{A}$ & LM & $\mathrm{B}$ & Cubic & 3 & 6 & 40 & 9 & 29 & 34 \\
\hline $\mathrm{A}$ & LM & $\mathrm{I}$ & Linear & $*$ & $*$ & $*$ & 9 & 90 & 799 \\
\hline A & LM & I & Cubic & $*$ & $*$ & $*$ & 9 & 102 & 6396 \\
\hline B & IR & $\bar{B}$ & Linear & 2 & 4 & 223 & 9 & 54 & 19 \\
\hline $\mathrm{B}$ & IR & $\mathrm{B}$ & Cubic & 2 & 4 & 168 & 8 & 16 & 9 \\
\hline B & IR & $\mathrm{I}$ & Linear & 2 & 4 & 358 & 9 & 54 & 398 \\
\hline B & IR & I & Cubic & $*$ & $*$ & $*$ & 9 & 54 & 2662 \\
\hline B & LM & B & Linear & 2 & 4 & 284 & 9 & 102 & 47 \\
\hline $\mathrm{B}$ & LM & $\mathrm{B}$ & Cubic & 2 & 4 & 188 & 9 & 18 & 34 \\
\hline $\mathrm{B}$ & LM & I & Linear & 2 & 4 & 474 & 9 & 102 & 914 \\
\hline $\mathrm{B}$ & LM & $\mathrm{I}$ & Cubic & $*$ & $*$ & $*$ & 9 & 102 & 4888 \\
\hline
\end{tabular}

Table 1: Here problem A has $D=.275, \mu=.018$ and problem B has $D=.275, \mu=.012$. The number of steps to converge to $5 \times 10^{-3}$ is given and $*$ indicates not converged to this tolerance in 9 steps. The total number of function evaluations (FE) is given in each case and also given are the equivalent results for convergence to $10^{-4}$ or the total number of function evaluations when convergence has not occured.

a total of 48 function evaluations. This suggests that condition (2.2) is indeed more severe than necessary, and in particular all the IRGN results are improved by allowing 5 backtracking steps. By considering only the tabulated results, one might conclude that there is no advantage to the use of IRGN methods as compared to LM, when regularization is applied in physical space, but it is clear from both Figures 1 and 2, that $D$ can be severely damped in the case with cubic splines, even if $\mu$ reaches an appropriate peak value. Moreover, in considering the number of steps to achieve a smaller residual, IRGN methods yield a smaller residual in fewer steps than with LM. In general the linear splines yield smoother results than cubic splines.

\section{Conclusions}

In this paper, we have established theoretical convergence results for an IRGN method in which stabilization is provided by a modified Tikhonov regularization term. Our theoretical results have been validated using simulations for a one dimensional version of the optical tomography inverse problem. We conclude from our simulations that the modified algorithm permits greater flexibility in regularizing GN iterations for solutions of ill-posed problems which naturally exhibit properties with different spatial scales, or for which known reference values are available in physical space. Moreover, mapping to physical space offers the advantage of determining a relevant constraining range for the B-spline coefficients. 


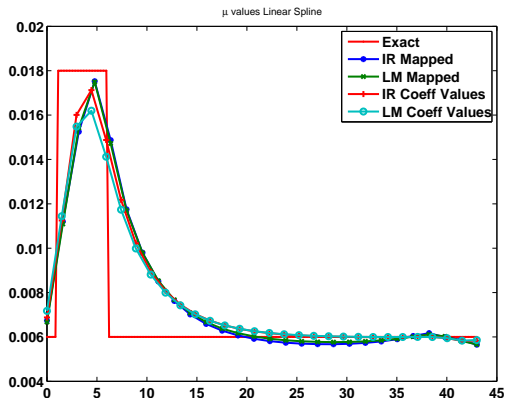

(a)

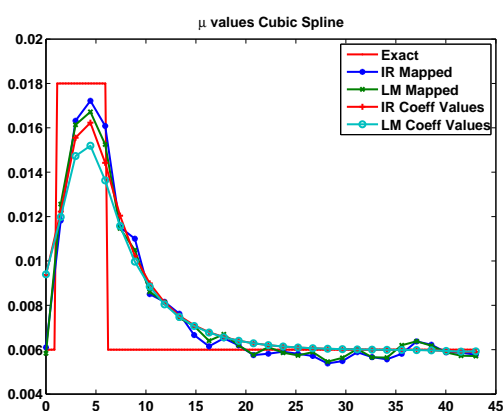

(c)

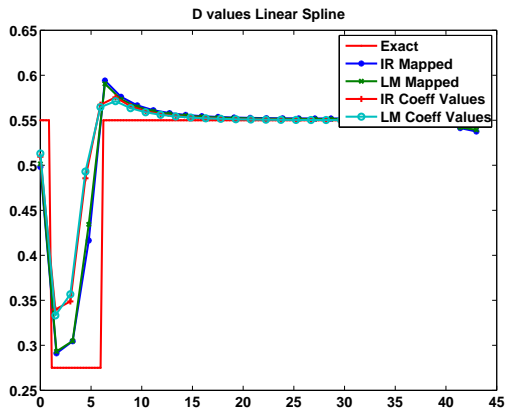

(e)

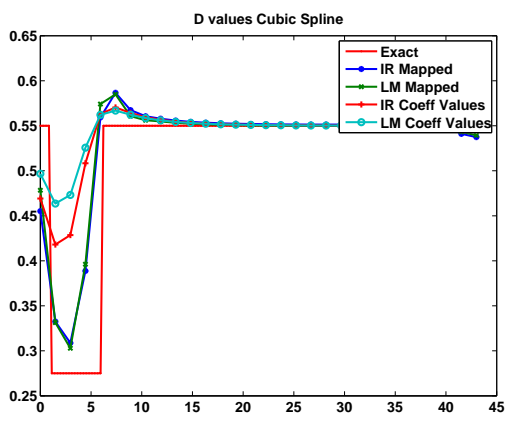

(g)

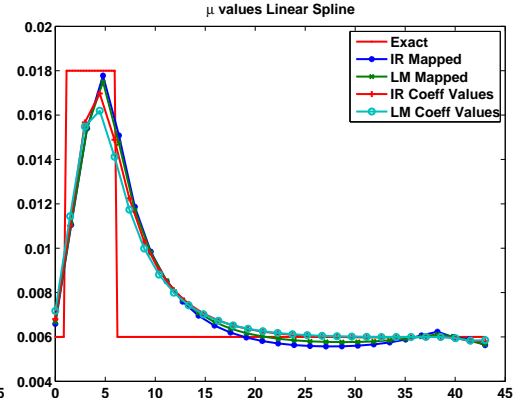

(b)

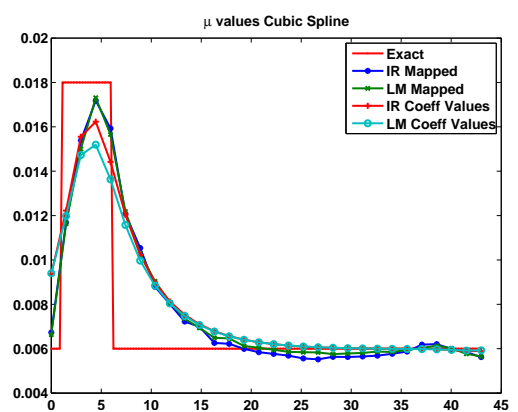

(d)

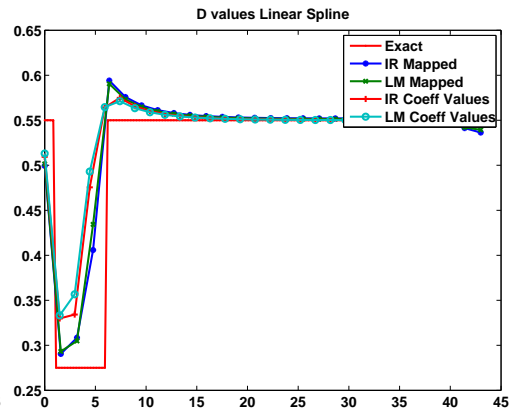

(f)

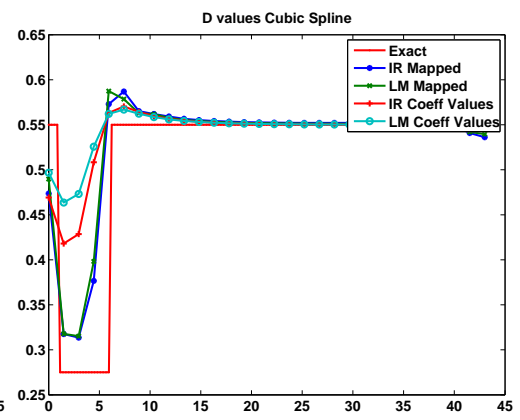

(h)

Figure 1: Problem A solved in (a,b,e,f) with linear splines and $(\mathrm{c}, \mathrm{d}, \mathrm{g}, \mathrm{h})$ with cubic splines. Results are shown at convergence to $.5 \times 10^{-4}$ on the left and $10^{-4}$ on the right, or at the $9^{\text {th }}$ iteration where convergence has not converged. 


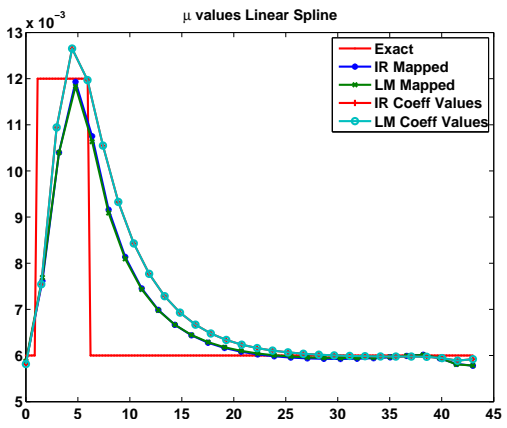

(a)

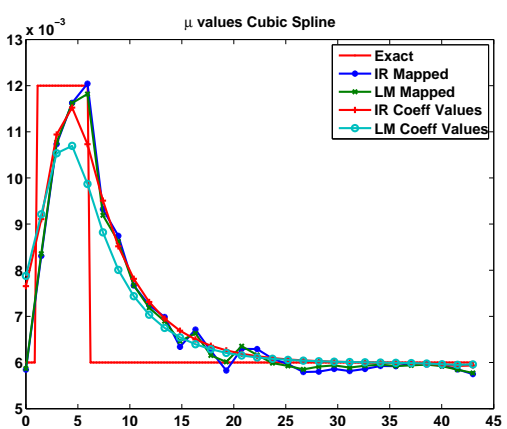

(c)

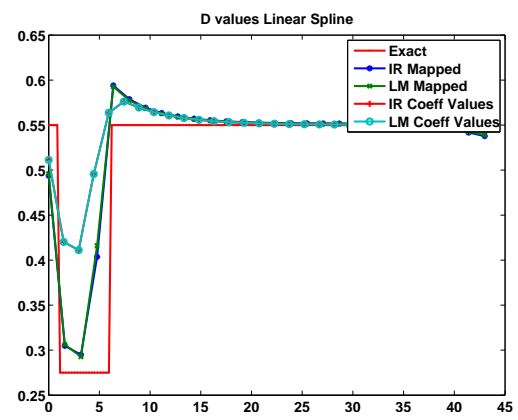

(e)

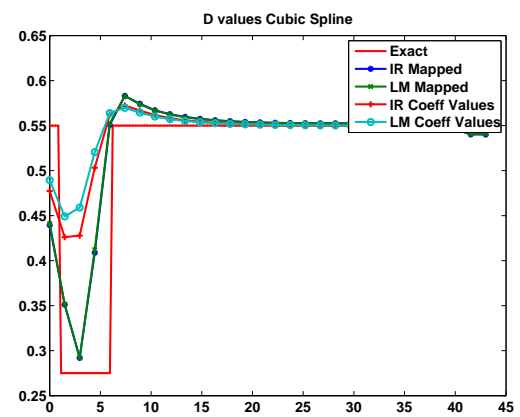

(g)

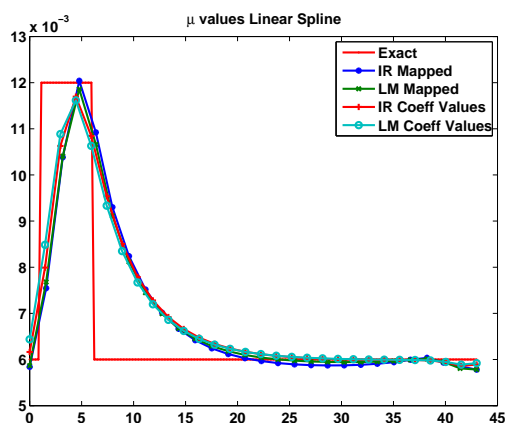

(b)

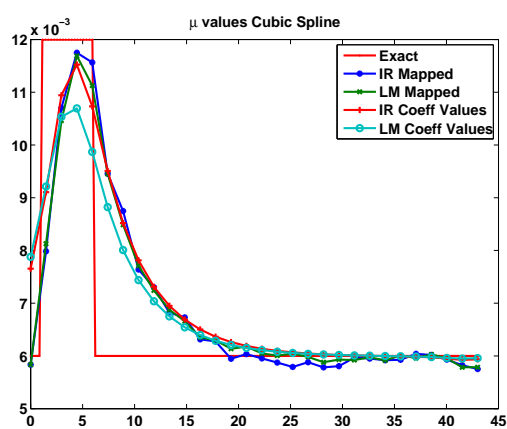

(d)

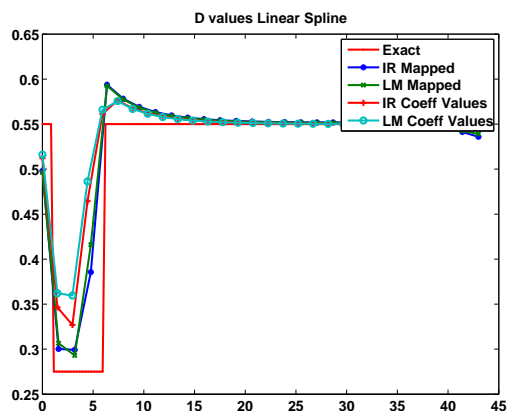

(f)

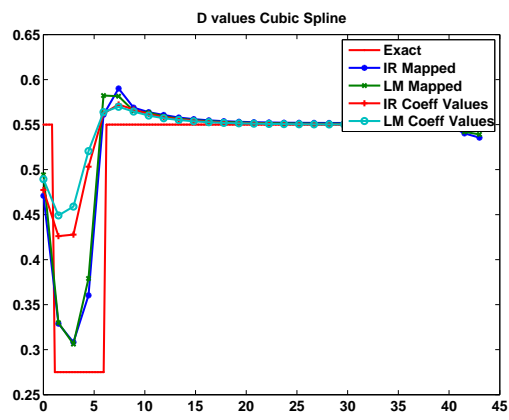

(h)

Figure 2: Problem B solved in (a,b,e,f) with linear splines and (c,d,g,h) with cubicsplines. Results are shown at convergence to $.5 \times 10^{-4}$ on the left and $10^{-4}$ below on the right, or at the $9^{\text {th }}$ iteration where convergence has not converged. 
SRK, February 13, 2007

\section{References}

[A99] S.R. Arridge, Optical tomography in medical imaging: Topical Review, Inverse Problem, 15 R41-R93, (1999).

[BA93] Bakushinsky, A. B., Iterative methods for nonlinear operator equations without regularity, new approach, Dokl. Russian Acad. Sci. 330 282-284, (1993).

[BA97] Bakushinsky, A. B., Iterative methods for the solution of nonlinear operator equations without the property of regularity. Fundamental and Applied Mathematics, 3, 685-692, (1997) (in Russian).

[BK01] Bakushinsky, A.B. and Kokurin, M.Yu. Sourcewise representation of solutions to nonlinear operator equations in a Banach space and convergence rate estimates for a regularized Newton's method, J. Inverse Ill-Posed Probl. 9 4, 349-374, (2001).

[BK04] Bakushinsky, A. B., Kokurin, M.Yu. Iterative methods for Ill-Posed Operator Equations with Smooth Operators, Springer Dordrecht, Great Britain, (2004).

[BS05] Bakushinsky, A. B. and Smirnova, A. On application of generalized discrepancy principle to iterative methods for nonlinear ill-posed problems, Numerical Functional Analysis and Optimization, 26, N1, 35-48, (2005).

[BKS05] Bakushinsky, A. B., Khan, T., and Smirnova, A. Inverse Problem in Optical Tomography and its Numerical Investigation by Iteratively Regularized Methods, Journal of Inverse and Ill-posed Problems, 13, 4, 1-14, (2005).

[BNS97] Blaschke B. (Kaltenbacher B.) and Neubauer A. and Scherzer O., On convergence rates for the iteratively regularized Gauss-Newton method, IMA J. Num. Anal., 17, 421-436, (1997).

[BKA06] Burger M., and Kaltenbacher B., Regularizing Newton-Kaczmarz Methods for Nonlinear Ill-Posed Problems, SIAM J. Num Anal., 44, 1, (2006) 153-182.

[CA93] B. Chance and R.R. Alfano, Photon migration and imaging in random media and tissues, SPIE 1888, (1993).

[CA95] B. Chance and R.R. Alfano, Optical tomography, photon migration, and spectroscopy of tissue and model media: theory, human studies, and instrumentations, part 1 and 2, SPIE 2389 (1995).

[DC97] D.T. Delphy and M. Cope, Quantification in tissue near-infrared spectroscopy, Phil. Trans. R. Soc. Lond. B 352 649-659, (1997).

[HAD97] J. Hebden, S.R. Arridge, and D.T. Delphy, Optical imaging in medicine: 1. Experimental techniques, Phys. Med. Biol. 42 825-840,(1997). 
[HAB98] A. Hielscher, R. Alcouffe, and R. Barbour, Comparison of finite-difference transport and diffusion calculations for photon migration in homogenous and heterogenous tissues, Phys. Med. Biol. 42 12851302, (1998).

[H97] T. Hohage, Logarithmic convergence rates of the iteratively regularized Gauss-Newton method for an inverse potential and inverse scattering problem, Inverse Problems, 13, 1279-1299, (1997).

[K00] Kaltenbacher, B., A projection-regularized Newton method for nonlinear ill-posed problems and its application to parameter identification problems with finite element discretizations, SIAM J. Num. Anal., 37, 6, 1885-1908, (2000).

[K99] Kelley, C. T., Iterative Methods for Optimization,SIAM, Frontiers in Applied Mathematics, (1999).

[KS05] Khan, T. and Smirnova, A., 1D inverse problem in diffusion based optical tomography using iteratively regularized Gauss-Newton algorithm, Applied Mathematics and Computation, Elsevier Science Inc., 161, 149-170, (2005).

[KW93] Kunisch K. and Ring N. Regularization of Nonlinear Illposed Problems with Closed Operators. Numerical Functional Analysis and Optimization, 14, 389-404, (1998).

[NW01] F. Natterer and F. Wubbeling, Mathematical Methods in Image Reconstruction, SIAM Monographs on Mathematical Modeling and Computation, (2001).

[NW99] Nocedal, J. \& Wright, S. J., Numerical Optimization, Springer-Verlag New York, (1999). 